\title{
Solutions of Partial Differential Equations with the CIP-BS Method*
}

\author{
Takayuki UTSUMI** and Hideo KIMURA***
}

\begin{abstract}
In this paper, we show that a new numerical method, the Constrained Interpolation Profile - Basis Set (CIP-BS) method, can solve partial differential equations (PDEs) with high accuracy and can be a universal solver by presenting examples for the solutions of typical parabolic, hyperbolic, and elliptic equations. Here, we present the numerical errors caused by this method, and illustrate that the solutions by the CIP-BS ${ }^{2}$ method, in which fifth order polynomials are used to constrain the values and first and second order spatial derivatives, are highly refined compared to those by the CIP-BS ${ }^{1}$ method, in which third order polynomials are used to constrain the values and first order spatial derivatives. The fact that this method can unambiguously solve PDEs with an one-to-one correspondence to analytical requirements is also shown for PDEs including singular functions like the Dirac delta function with Dirichet or Neumann boundary conditions. This method is straightforwardly applicable to PDEs describing complex physical and engineering problems.
\end{abstract}

Key Words: Numerical Analysis, Numerical Technique, Partial Differential Equation, The CIP-BS Method, Error Evaluation

\section{Introduction}

The role of numerical simulations is now becoming increasingly important in the analysis of complex systems described by various types of partial differential equations (PDEs). Much effort and investment has been devoted to improving the accuracy and stability of numerical solutions. We can expect that the recent progress in the computer hardware will resolve these issues to some extent by enabling the use of finer meshes. However, nowadays, it is widely recognized that tracing sharp discontinuities of physical quantities such as shock waves or interfaces between different materials can not be satisfactorily accomplished with this technique. Therefore, there is continuing interest in developing a more robust and efficient universal method for numerically solving PDEs with high accuracy and stability.

Although nature operates in a continuous world, dis-

* Received 8th April, 2004 (No. 04-4084)

** Advanced Photon Research Center, Japan Atomic Energy Research Institute, 8-1 Umemidai, Kizu-cho, Soraku-gun, Kyoto-fu 619-0215, Japan.

E-mail: utsumi@apr.jaeri.go.jp

*** Center for Promotion of Computational Science and Engineering, Japan Atomic Energy Research Institute, 6-9-3 Higashiueno, Taito-ku, Tokyo 110-0015, Japan cretization is unavoidable for implementing numerical simulations. Therefore, the most important point for the numerical method will be how to retrieve the information lost inside the grid cell between the discretized points. The Constrained Interpolation Profile (CIP) method ${ }^{(1),(2)}$ proposed by $\mathrm{T}$. Yabe et. al. introduced a Hermite type interpolation function whose coefficients are determined not only by functional values but also their first derivatives consistent with the governing equations. This means that derivatives are also independent variables associated with the grid point, and obey equations derived by taking derivatives of the governing equations. The CIP method has been shown to be a low numerical diffusion and stable method as a universal solver for hyperbolic differential equations, and has been successfully applied to various complex linear and nonlinear hydrodynamic problems, covering both compressible and incompressible flow, such as shock wave generation, laser-induced evaporation, and elastic-plastic flow ${ }^{(3)-(7)}$.

It is worth noting that the CIP method does not include any algorithm-dependent parameter. If the size of the system and grid intervals for the problem are defined, the governing equations are uniquely transformed into a discretized form, in which no matrix solution is used. However, methods using matrix operations are more advantageous because a number of numerical methods for 
large, sparse linear systems developed for the finite difference method or the finite element method can be adopted. Recently, a new numerical method, the CIP-Basis Set (CIP-BS) method ${ }^{(8)}$, has been proposed by generalizing the concept of the CIP method from the viewpoint of the basis set. The governing equations are unambiguously discretized into matrix form equations requiring the residuals to be orthogonal to the basis functions via the same procedure as the Galerkin method. The CIP-BS method, in which the local polynomial basis functions corresponding to the values and spatial derivatives at each grid point belong to the complete set and the $\mathrm{C}^{K}$ class, is called the CIP-BS ${ }^{K}$ method. Numerical results in the solution of the Schrödinger equation have demonstrated that accurate solutions are obtained by the CIP-BS method and the use of a higher order basis set is essential in increasing accuracy.

In this paper, we show that the CIP-BS method can solve various types of PDEs with high accuracy and be a universal solver for them by presenting examples for the solutions of typical parabolic, hyperbolic, and elliptic equations. The fact that this method can unambiguously solve PDEs with a one-to-one correspondence to analytical requirements is also shown for equations including singular functions like the Dirac delta function with Dirichet or Neumann boundary conditions.

The paper is organized as follows: In section 2, we briefly review the CIP-BS method and define the PDEs to be solved, i.e. the thermal diffusion, the advection, and the Poisson equation. Numerical results, in particular those produced by the CIP-BS ${ }^{1}$ and CIP-BS ${ }^{2}$ method, are shown in section 3. Conservation and the order of numerical errors are also discussed. Concluding remarks are given in section 4, emphasizing the computational efficiency and applicability of the CIP-BS method for the study of the dynamics of a broad spectrum of complex physical and engineering problems.

\section{Numerical Methods}

\subsection{The CIP-BS method}

Since the CIP-BS method is new and not widely known, we first summarize the method.

We define the values and derivatives of an arbitrary function $f(x)$ at the grid points, and assume that the functions in the calculation domain can be approximated by the CIP-basis set of degree $K$, where $K$ refers to the order of the derivatives we retain in the calculation. The expression for this function is

$$
f(x)=\sum_{i=1}^{N} \sum_{k=0}^{K} f_{i}^{(k)} \phi_{k, i}(x),
$$

where $f_{i}^{(k)}$ is the $k$-th coefficient at the grid point $x_{i}$, the summation on the index $i$ is taken over all grid points, and the basis functions $\phi_{k, i}(x)$ with local support $\left[x_{i-1}, x_{i+1}\right]$ are expressed in the form

$$
\begin{gathered}
\phi_{k, i}(x)=\left(\theta\left(x-x_{i-1}\right)-\theta\left(x-x_{i}\right)\right) \phi_{k, i-}(x) \\
\quad+\left(\theta\left(x-x_{i}\right)-\theta\left(x-x_{i+1}\right)\right) \phi_{k, i+}(x),
\end{gathered}
$$

where $\theta(x)$ is the Heaviside step function and $\phi_{k, i \pm}(x)$ are polynomials of degree $(2 K+1)$ determined from the constraints at the grid points

$$
\begin{aligned}
& D_{x}^{l} \phi_{k, i \pm}\left(x_{i}\right)=\left\{\begin{array}{l}
1 \text { for } l=k \\
0 \text { for } l=0,1, \ldots, k-1, k+1, \ldots, K
\end{array}\right. \\
& D_{x}^{l} \phi_{k, i \pm}\left(x_{i \pm 1}\right)=0 \text { for } 0,1, \ldots, K,
\end{aligned}
$$

where $D_{x}$ is a partial differential operator $\left(D_{x}=\partial / \partial x\right)$, $D_{x}^{0}=1$.

The polynomials $\phi_{k, i \pm}$ in the basis functions for the CIP-BS $^{0}$ method are

$$
\begin{aligned}
& \phi_{0, i-}(x)=1+\bar{x} / \Delta x_{i-}, \\
& \phi_{0, i+}(x)=1-\bar{x} / \Delta x_{i+},
\end{aligned}
$$

and those for the CIP-BS ${ }^{1}$ method are

$$
\begin{aligned}
& \phi_{0, i-}(x)=1-3 \bar{x}^{2} / \Delta x_{i-}^{2}-2 \bar{x}^{3} / \Delta x_{i-}^{3}, \\
& \phi_{0, i+}(x)=1-3 \bar{x}^{2} / \Delta x_{i+}^{2}+2 \bar{x}^{3} / \Delta x_{i+}^{3}, \\
& \phi_{1, i-}(x)=-\bar{x}+2 \bar{x}^{2} / \Delta x_{i-}-\bar{x}^{3} / \Delta x_{i-}^{2}, \\
& \phi_{1, i+}(x)=\bar{x}-2 \bar{x}^{2} / \Delta x_{i+}-\bar{x}^{3} / \Delta x_{i-}^{2},
\end{aligned}
$$

and those for the CIP-BS ${ }^{2}$ method are

$$
\begin{aligned}
& \phi_{0, i-}(x)=1+10 \bar{x}^{3} / \Delta x_{i-}^{3}+15 \bar{x}^{4} / \Delta x_{i-}^{4}+6 \bar{x}^{5} / \Delta x_{i-}^{5}, \\
& \phi_{0, i+}(x)=1-10 \bar{x}^{3} / \Delta x_{i+}^{3}+15 \bar{x}^{4} / \Delta x_{i+}^{4}-6 \bar{x}^{5} / \Delta x_{i+}^{5}, \\
& \phi_{1, i-}(x)=\bar{x}-6 \bar{x}^{3} / \Delta x_{i-}^{2}-8 \bar{x}^{4} / \Delta x_{i-}^{3}-3 \bar{x}^{5} / \Delta x_{i-}^{4}, \\
& \phi_{1, i+}(x)=\bar{x}-6 \bar{x}^{3} / \Delta x_{i+}^{2}+8 \bar{x}^{4} / \Delta x_{i+}^{3}-3 \bar{x}^{5} / \Delta x_{i+}^{4}, \\
& \phi_{2, i-}(x)=\bar{x}^{2} / 2+3 \bar{x}^{3} / 2 \Delta x_{i-}+3 \bar{x}^{4} / 2 \Delta x_{i-}^{2}+\bar{x}^{5} / 2 \Delta x_{i-}^{3}, \\
& \phi_{2, i+}(x)=\bar{x}^{2} / 2-3 \bar{x}^{3} / 2 \Delta x_{i+}+3 \bar{x}^{4} / 2 \Delta x_{i+}^{2}-\bar{x}^{5} / 2 \Delta x_{i+}^{3},
\end{aligned}
$$

where $\bar{x}=x-x_{i}, \Delta x_{i-}=x_{i}-x_{i-1}, \Delta x_{i+}=x_{i+1}-x_{i}$. In particular, for a uniform grid system $\left(\Delta x_{i-}=\Delta x_{i+}=\Delta x\right)$ the basis function satisfies the translational relation

$$
\phi_{k, i}(x)=\phi_{k, i-n}\left(x-x_{n}\right) .
$$

From this definition, we can easily verify that the expansion function Eq. (1) for the CIP-BS ${ }^{0}$, CIP-BS ${ }^{1}$, and CIP$\mathrm{BS}^{2}$ method corresponds to the linear, CIP, and IDO $^{(9)}$ interpolation function, respectively. We introduce the scalar product of the function $g(x)$ and $h(x)$ in the domain $R$ as

$$
<g \mid h>=\int_{R} \bar{g}(x) h(x) d x,
$$

where $\bar{g}(x)$ is the complex conjugate of $g(x)$. Since the support of $\phi_{i}(x)$ is $\left[x_{i-1}, x_{i+1}\right]$, the scalar product and matrix elements of the spatial differential operator $D_{x}^{n}$ can be analytically calculated as shown in the Appendix.

The PDEs $\partial f / \partial t=L[f]$ or $g=L[f]$, where $g$ is a known function, are discretized and reduced to matrix equations by the scalar products with basis functions $\phi_{k, i}$. When $L$ is a linear operator, we obtain the matrix equation $S d f / d t=L f$ or $L f=S \boldsymbol{g}$, where $S$ is a positive definite 
matrix with the element $S_{k i, k^{\prime} i^{\prime}}$. Since $S_{k i, k^{\prime} i^{\prime}}$ is non-zero only for $i=i^{\prime}-1, i^{\prime}, i^{\prime}+1, S$ is a band diagonal matrix with bandwidth $3(K+1)$. The matrix representation of the operator $L$ also has the same structure as the matrix $S$, and the matrix elements $<\phi_{k, i}|L| \phi_{k^{\prime} i^{\prime}}>$ can be analytically calculated. It is emphasized that the only spatial discretization is performed in the CIP-BS method. Therefore, the timedependent problems expressed by the ordinary differential equations, $S d f / d t=L f$, must be solved by means of either implicit or explicit time propagation scheme $\mathrm{e}^{(10),(11)}$.

Next, we define the typical PDEs solved to demonstrate the efficiency of the CIP-BS method.

\subsection{Thermal diffusion equation}

The typical parabolic PDE we solve is the onedimensional thermal diffusion equation written as

$$
\frac{\partial f}{\partial t}=\kappa \frac{\partial^{2} f}{\partial x^{2}},
$$

where $f$ is the temperature and $\kappa$ is the thermal conductivity.

\subsection{Advection equation}

The typical hyperbolic PDE we solve is the onedimensional advection equation written as

$$
\frac{\partial f}{\partial t}+u \frac{\partial f}{\partial x}=0
$$

where $f$ is the profile and $u$ is the flow speed. In a real flow, the flow velocity depends on the position $x$, however, here we assume $u$ is constant at any point $x$ for simplicity.

\subsection{Poisson equation}

The typical elliptic PDE we solve is the Poisson equation written as

$$
\frac{\partial^{2} f}{\partial x^{2}}=g(x)
$$

where $f$ is the potential to be solved and $g(x)$ is some known function.

In this paper, we solve two types of Poisson equations

$$
\frac{\partial^{2} f}{\partial x^{2}}=\cos (x),
$$

and

$$
\frac{\partial^{2} f}{\partial x^{2}}=\delta(x)
$$

where $\delta(x)$ is the Dirac delta function. Although Eq. (13) contains a singular function, the CIP-BS method can properly discretize it due to the use of the scalar product between $\delta(x)$ and the basis function.

\section{Numerical Results}

\subsection{Thermal diffusion equation}

We have solved Eq. (9) with the thermal conductivity coefficient $\kappa=0.01$ in the region $[0,1]$ for the time $[0,30]$. The initial profile is $\exp (\cos (\pi x)-1) / 2 \kappa \pi)$ and the boundary condition is $f^{\prime}(0)=f^{\prime}(1)=0$. Since the discretized ordinary differential equations are stiff in many cases, we used an implicit solver with general sparse Jacobian matrices to time propagate the solution developed

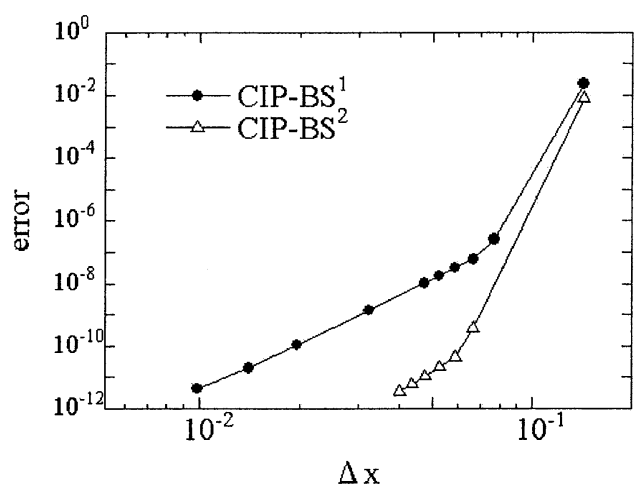

Fig. 1 The deviation from the exact solution versus the grid interval for the solution of the thermal equation by the CIP-BS $^{1}$ and CIP-BS ${ }^{2}$ method

by Hindmarsh and Balsdon ${ }^{(11)}$. Figure 1 shows relative errors as a function of the grid size $\Delta x$ at $t=30$. Relative error is defined as the sum of the difference between the exact(analytic) value and the calculated value for all grid points divided by a sum of the exact value for all grid points,

$$
\text { error }=\frac{\sum_{i=1}^{N}\left|f_{\text {exac }, i}(t)-f_{\text {calc }, i}(t)\right|}{\sum_{i=1}^{N}\left|f_{\text {exac }, i}(t)\right|},
$$

where $f_{\text {exac }, i}(t)$ is the exact value at the grid point $i$ at the time $t, f_{\text {calc }, i}(t)$ is the calculated value, and $N$ is the total number of grid points.

In Fig. 1, it is recognized that the accuracy of the CIP$\mathrm{BS}^{1}$ method is roughly the same as that of the CIP-BS ${ }^{2}$ method when the grid size is large $\left(\Delta x>10^{-1}\right)$ where the initial profile might not be precisely recognized due to the lack of grid points. However for smaller grid size, the CIP$\mathrm{BS}^{1}$ method has about fourth order accuracy and the CIP$\mathrm{BS}^{2}$ method possesses about sixth order accuracy. The discretized equation of Eq. (9) by the CIP-BS ${ }^{1}$ method, is written as

$$
\begin{gathered}
\frac{9 \Delta x}{70} \dot{f}_{n-1}^{0}+\frac{26 \Delta x}{35} \dot{f}_{n}^{0}+\frac{9 \Delta x}{70} \dot{f}_{n+1}^{0}+\frac{13 \Delta x^{2}}{420} \dot{f}_{n-1}^{1}-\frac{13 \Delta x^{2}}{420} \dot{f}_{n+1}^{1} \\
=\kappa\left(\frac{6}{5 \Delta x} f_{n-1}^{0}-\frac{12}{5 \Delta x} f_{n}^{0}+\frac{6}{5 \Delta x} f_{n+1}^{0}+\frac{1}{10} f_{n-1}^{1}-\frac{1}{10} f_{n+1}^{1}\right), \\
-\frac{13 \Delta x^{2}}{420} \dot{f}_{n-1}^{0}+\frac{13 \Delta x^{2}}{420} \dot{f}_{n+1}^{0}-\frac{\Delta x^{3}}{140} \dot{f}_{n-1}^{1}+\frac{2 \Delta x^{3}}{105} \dot{f}_{n}^{1}-\frac{\Delta x^{3}}{140} \dot{f}_{n+1}^{1} \\
=\kappa\left(-\frac{1}{10} f_{n-1}^{0}+\frac{1}{10} f_{n+1}^{0}+\frac{\Delta x}{30} f_{n-1}^{1}-\frac{4 \Delta x}{15} f_{n}^{1}+\frac{\Delta x}{30} f_{n+1}^{1}\right),
\end{gathered}
$$

where $f_{1}^{1}=f_{N}^{1}=0$ due to the boundary condition, $f_{0}^{k}=f_{N+1}^{k}=0$, and the coefficients corresponding to $<\phi_{k, 1}\left|\phi_{k, 1}\right\rangle,\left\langle\phi_{k, N}\right| \phi_{k, N}>$ are multiplied by $1 / 2$ because the domain $R$ is $\left[x_{1}, x_{N}\right]$. By Substituting Taylor expansions of $f(x)$ into Eq. (15), we obtain the following two equations

$$
\frac{d}{d t}\left(f+\frac{1}{15} f^{\prime \prime} \Delta x^{2}+\frac{1}{2520} f^{(4)} \Delta x^{4}+O\left(\Delta x^{6}\right)\right)
$$




$$
\begin{gathered}
=\kappa\left(f^{\prime \prime}+\frac{1}{15} f^{(4)} \Delta x^{2}+\frac{1}{600} f^{(6)} \Delta x^{4}+O\left(\Delta x^{6}\right)\right), \\
\frac{d}{d t}\left(f^{\prime}+\frac{1}{21} f^{(3)} \Delta x^{2}-\frac{1}{840} f^{(5)} \Delta x^{4}+O\left(\Delta x^{6}\right)\right) \\
=\kappa\left(f^{(3)}+\frac{1}{15} f^{(5)} \Delta x^{2}+\frac{1}{504} f^{(7)} \Delta x^{4}+O\left(\Delta x^{6}\right)\right) .
\end{gathered}
$$

In the same way, we obtain three equations for the CIP$\mathrm{BS}^{2}$ method as

$$
\begin{aligned}
& \frac{d}{d t}\left(f+\frac{5}{84} f^{\prime \prime} \Delta x^{2}+\frac{1}{720} f^{(4)} \Delta x^{4}+\frac{31}{1108800} f^{(4)} \Delta x^{6}\right. \\
& \left.\quad+O\left(\Delta x^{8}\right)\right)=\kappa\left(f^{\prime \prime}+\frac{5}{84} f^{(4)} \Delta x^{2}+\frac{1}{720} f^{(6)} \Delta x^{4}\right. \\
& \left.\quad+\frac{1}{52920} f^{(8)} \Delta x^{6}+O\left(\Delta x^{8}\right)\right), \\
& \frac{d}{d t}\left(f^{\prime}+\frac{1}{24} f^{(3)} \Delta x^{2}+\frac{1}{1320} f^{(5)} \Delta x^{4}+\frac{17}{665280} f^{(7)} \Delta x^{6}\right. \\
& \left.\quad+O\left(\Delta x^{8}\right)\right)=\kappa\left(f^{(3)}+\frac{1}{24} f^{(5)} \Delta x^{2}+\frac{1}{1680} f^{(7)} \Delta x^{4}\right. \\
& \left.\quad+O\left(\Delta x^{8}\right)\right), \\
& \frac{d}{d t}\left(f+\frac{3}{28} f^{\prime \prime} \Delta x^{2}+\frac{1}{336} f^{(4)} \Delta x^{4}+\frac{19}{332640} f^{(6)} \Delta x^{6}\right. \\
& \left.\quad+O\left(\Delta x^{8}\right)\right)=\kappa\left(f^{\prime \prime}+\frac{3}{28} f^{(4)} \Delta x^{2}+\frac{1}{360} f^{(6)} \Delta x^{4}\right. \\
& \left.\quad+\frac{1}{60480} f^{(8)} \Delta x^{6}+O\left(\Delta x^{8}\right)\right) .
\end{aligned}
$$

Comparing Eqs. (16) and (17) with Eq. (9), we can see that the discretized equation contains some extra terms which do not emerge in the original equation. These extra terms might cause numerical errors. Equations (16) and (17) are polynomial functions of $\Delta x$, and we can see that the first and second terms on both sides of Eq. (16) are almost the same form. Beyond the third term which is a polynomial of order $\Delta x^{4}$ the terms differ. In Eq. (17), the first three terms are of almost the same form and beyond the fourth term which is polynomial of $\Delta x^{6}$ they differ. Therefore, if we consider Eq. (16) as function of the form $u=f+A f^{\prime \prime} \Delta x^{2}(A$ is constant $)$, it has fourth order accuracy, $\frac{d u}{d t}=\kappa \frac{\partial^{2} u}{\partial x^{2}}+O\left(\Delta x^{4}\right)$. And if we consider Eq. (17) as function of the form $u=f+A f^{\prime \prime} \Delta x^{2}+B f^{(4)} \Delta x^{4}(A$ and $B$ are constants), it has sixth order accuracy, $\frac{d u}{d t}=\kappa \frac{\partial^{2} u}{\partial x^{2}}+O\left(\Delta x^{6}\right)$.

By taking sum of both sides of Eq. (15), we obtain $\frac{d}{d t}\left(\frac{1}{2} f_{1}^{0}+\sum_{i=2}^{N-1} f_{i}^{0}+\frac{1}{2} f_{N}^{0}\right)=\frac{d}{d t} \int_{R} f(x) d x=0$. In the same procedure, $\frac{d}{d t}\left(\left(\frac{1}{2} f_{1}^{0}+\sum_{i=2}^{N-1} f_{i}^{0}+\frac{1}{2} f_{N}^{0}\right)+\frac{\Delta x^{2}}{60}\left(\frac{1}{2} f_{1}^{2}+\sum_{i=2}^{N-1} f_{i}^{2}+\right.\right.$ $\left.\left.\frac{1}{2} f_{N}^{2}\right)\right)=\frac{d}{d t} \int_{R} f(x) d x=0$ is derived for the CIP-BS ${ }^{2}$ method. We can see that the total heat is conserved in principle for the CIP-BS ${ }^{1}$ and CIP-BS ${ }^{2}$ method.

\subsection{Advection equation}

The advection equation is solved with the velocity $u=0.05$ in the region $[0,1]$ for the time $[0,20]$. We have solved for two initial conditions; one is a sine wave $(\sin (2 \pi x)[0 \leq x \leq 1])$ and the other is a square wave $(f(x)=$
$1[0.45 \leq x \leq 0.55], f(x)=0[0 \leq x<0.45$ and $0.55<x \leq 1])$. A periodic boundary condition is imposed as $f(0)=f(1)$. It is noted that we set $f_{i}^{k}=0(k \geq 1, i=1,2, \cdots, N)$ for a initial square wave approximating the sharp edge by the function $f_{i}^{0} \phi_{0, i+}$ or $f_{i}^{0} \phi_{0, i-}$.

Figure 2 shows relative errors in solving the advection equation for a sine wave. This result shows fourth order accuracy for the CIP-BS ${ }^{1}$ method and sixth order accuracy for the CIP-BS ${ }^{2}$ method. These accuracies are accounted for in the same manner described in the case of the thermal diffusion equation.

The numerical solutions of the advection equation for a square wave are shown in Fig. 3. Since the analytical solution is $f(x-u t)=$ const, the profile is shifted and returns to the initial position at $t=20$ due to the periodic boundary condition. Whereas there is slight leaning at the wavetop, the shape is conserved. The conservation property is proved for the periodic boundary condition by taking sum of both sides of Eq. (18) over all grid points. Since the discretized equation of Eq. (10) for the CIP-BS ${ }^{1}$ method is written as

$$
\begin{gathered}
\frac{9 \Delta x}{70} \dot{f}_{n-1}^{0}+\frac{26 \Delta x}{35} \dot{f}_{n}^{0}+\frac{9 \Delta x}{70} \dot{f}_{n+1}^{0}+\frac{13 \Delta x^{2}}{420} \dot{f}_{n-1}^{1}-\frac{13 \Delta x^{2}}{420} \dot{f}_{n+1}^{1} \\
=-\frac{1}{2} f_{n-1}^{0}+\frac{1}{2} f_{n+1}^{0}-\frac{\Delta x}{10} f_{n-1}^{1}+\frac{\Delta x}{5} f_{n}^{1}-\frac{\Delta x}{10} f_{n+1}^{1}
\end{gathered}
$$

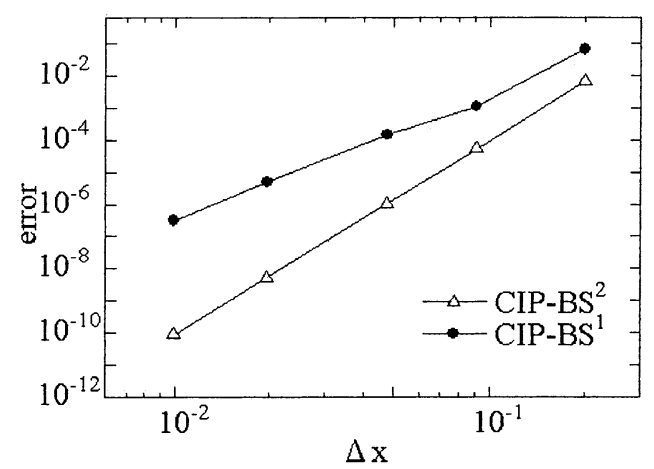

Fig. 2 The deviation from the exact solution versus the grid interval for the solution of the advection equation for a sine wave by the CIP-BS ${ }^{1}$ and CIP-BS ${ }^{2}$ method

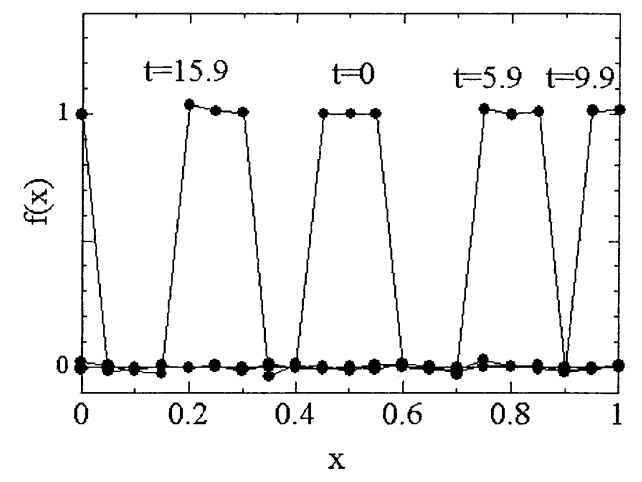

Fig. 3 Time evolution of a square wave. The advection equation is solved by the CIP-BS ${ }^{2}$ method with 21 grid points. 


$$
\begin{gathered}
-\frac{13 \Delta x^{2}}{420} \dot{f}_{n-1}^{0}+\frac{13 \Delta x^{2}}{420} \dot{f}_{n+1}^{0}-\frac{\Delta x^{3}}{140} \dot{f}_{n-1}^{1}+\frac{2 \Delta x^{3}}{105} \dot{f}_{n}^{1}-\frac{\Delta x^{3}}{140} \dot{f}_{n+1}^{1} \\
=\frac{\Delta x}{10} f_{n-1}^{0}-\frac{\Delta x}{5} f_{n}^{0}+\frac{\Delta x}{10} f_{n+1}^{0}+\frac{\Delta x^{2}}{60} f_{n-1}^{1}-\frac{\Delta x^{2}}{60} f_{n+1}^{1},
\end{gathered}
$$

where $f_{0}^{k}=f_{N-1}^{k}$ and $f_{N+1}^{k}=f_{2}^{k}$ due to the periodic boundary condition, we can easily obtain

$$
\begin{aligned}
& \frac{d}{d t} \sum_{i} f_{i}^{0}=0, \\
& \frac{d}{d t} \sum_{i} f_{i}^{1}=0 .
\end{aligned}
$$

In the same manner, we obtain the following relations for the CIP-BS ${ }^{2}$ method

$$
\begin{aligned}
& \frac{d}{d t} \sum_{i}\left(f_{i}^{0}+\frac{\Delta x^{2}}{60} f_{i}^{2}\right)=0, \\
& \frac{d}{d t} \sum_{i} f_{i}^{1}=-\frac{33 u}{55} \sum_{i} f_{i}^{2}, \\
& \frac{d}{d t} \sum_{i}\left(f_{i}^{0}+\frac{\Delta x^{2}}{42} f_{i}^{2}\right)=\frac{3 u}{7} \sum_{i} f_{i}^{1} .
\end{aligned}
$$

Apparently $\sum_{i} f_{i}^{0}=\int_{R} f(x) d x, \sum_{i} f_{i}^{1}$ are conserved by the CIP-BS ${ }^{1}$ method. Since the equation $\frac{d^{2}}{d t^{2}} \sum_{i} f_{i}^{1}=$ $-36 u^{2} \sum_{i} f_{i}^{1}$ is derived from Eq. (20) and $\sum_{i} f_{i}^{1}=0$ at $t=0$ due to the periodic boundary condition, we can confirm that not only $\sum_{i}\left(f_{i}^{0}+\frac{\Delta x^{2}}{60} f_{i}^{2}\right)=\int_{R} f(x) d x$ but also $\sum_{i} f_{i}^{0}$ are conserved by the CIP-BS ${ }^{2}$ method. These quantities are conserved in principle, and the degree of conservation depends on the accuracy of the integration method we adopt. Figure 4 shows the time evolution of $\sum f_{i}^{0}$ where the relative and absolute tolerance are set to $10^{-12}$ for the time integration routine $L S O D I S^{(11)}$.

Figure 5 shows profiles at $t=20$. We can see that the numerical oscillations around the edges of a square wave are suppressed when we adopt higher order basis functions.

\subsection{Poisson equation}

We have solved Eqs. (12) and (13) in the region $[-1,1]$ with the boundary condition $f(-1)=f(1)=0$.

From Fig. 6, we recognize the CIP-BS ${ }^{1}$ method is about fourth order accuracy and about sixth order accuracy by the CIP-BS ${ }^{2}$ method in solving Eq. (12), i.e. when $g$ is a smooth function. On the other hand, the analytical solution of Eq. (13) is $f(x)=-\frac{1}{2}-\frac{1}{2} x+x \theta(x)$, and the numerical one is shown in Fig. 7. Figure 7 (c) shows both the CIP-BS ${ }^{1}$ and CIP-BS ${ }^{2}$ method have second order accuracy. From Fig. 7 (a) and (b), we can see that there are errors at the origin where the first derivative of the analytical solution is not continuous. The maximum error is decreased from $10^{-2}$ to $10^{-4}$ when the number of grid points is increased from 11 to 101 . In addition errors neighboring the

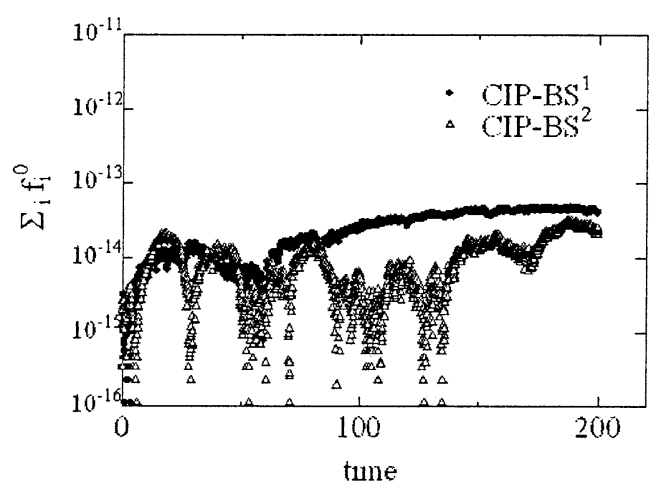

Fig. 4 Time evolution of $\sum f_{i}^{0}$ for the advection of a sine wave $(\sin (2 \pi x)[0 \leq x \leq 1])$ by the CIP-BS ${ }^{1}$ and CIP-BS ${ }^{2}$ method with 21 grid points

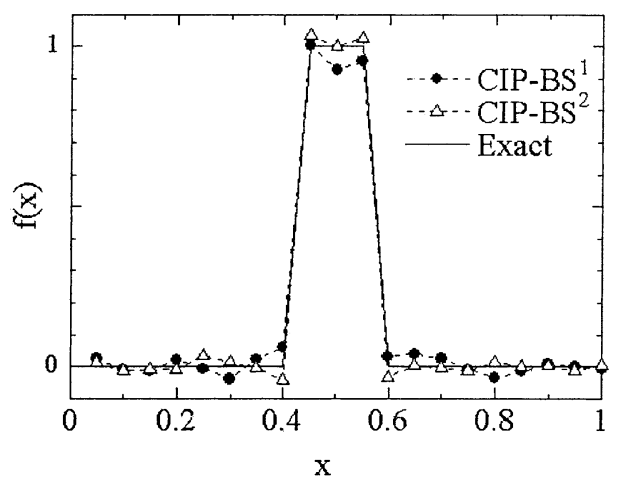

Fig. 5 Transport of a square wave by the advection equation computed by the CIP-BS ${ }^{1}$ and CIP-BS ${ }^{2}$ method with 21 grid points. Plotted are the numerical results at $t=20$ with $u=0.05$.

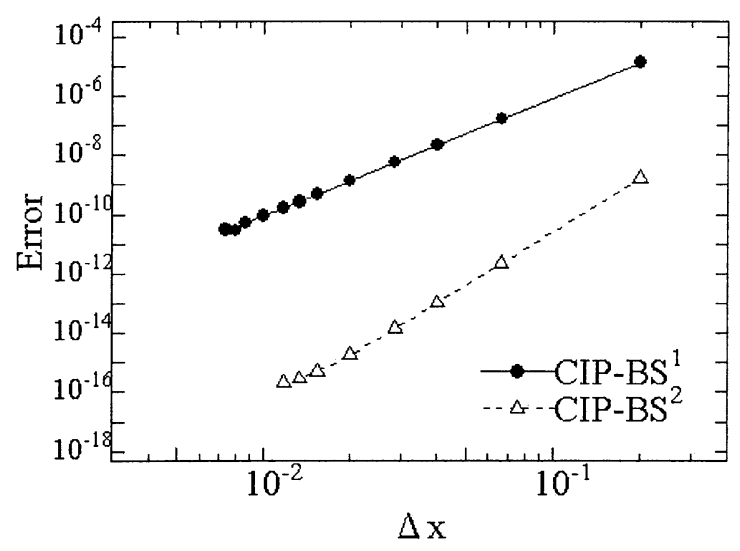

Fig. 6 The deviation from the exact solution versus the grid interval in solving Poisson equations $\frac{\partial^{2} f}{\partial x^{2}}=\cos (x)$, by the CIP-BS ${ }^{1}$ and CIP-BS ${ }^{2}$ method

origin are reduced exponentially out to the edges. As indicated in Fig. 7 (b), errors saturate at the machine accuracy $10^{-16}$.

This is justified by Taylor series expanding the discretized form of Eq. (11),

$$
\frac{6}{5 \Delta x} f_{n-1}^{0}-\frac{12}{5 \Delta x} f_{n}^{0}+\frac{6}{5 \Delta x} f_{n+1}^{0}+\frac{1}{10} f_{n-1}^{1}-\frac{1}{10} f_{n+1}^{1}
$$




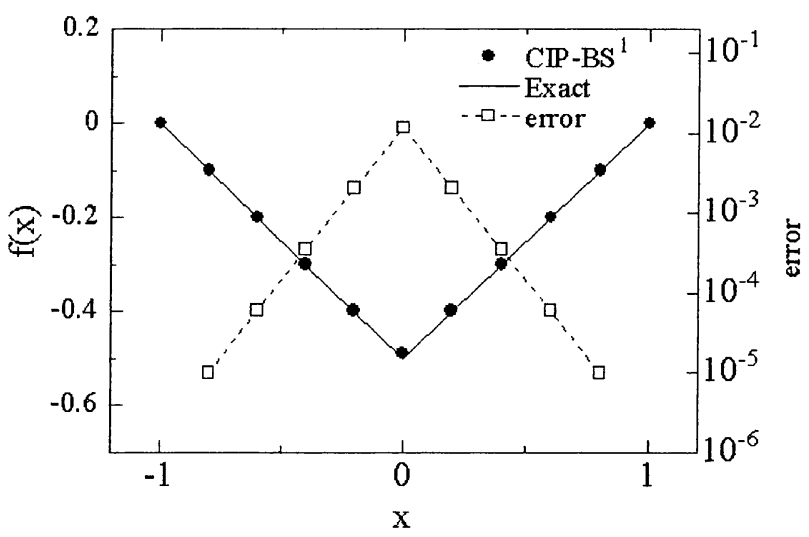

(a)

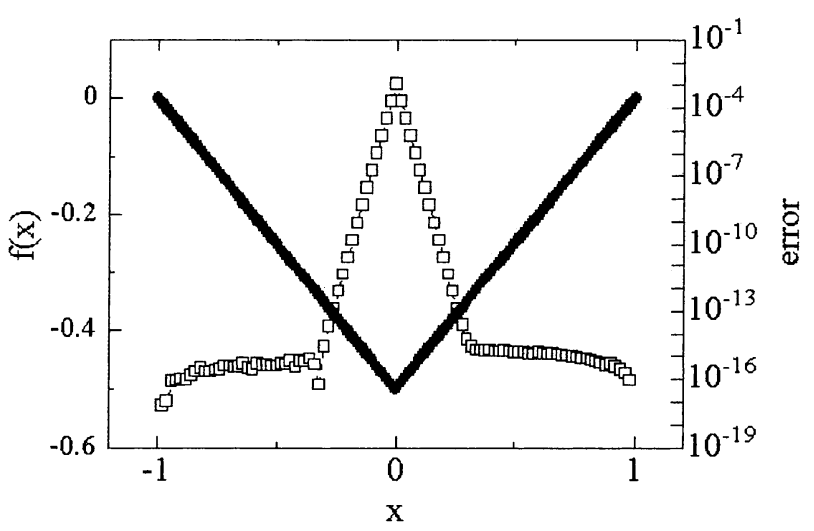

(b)

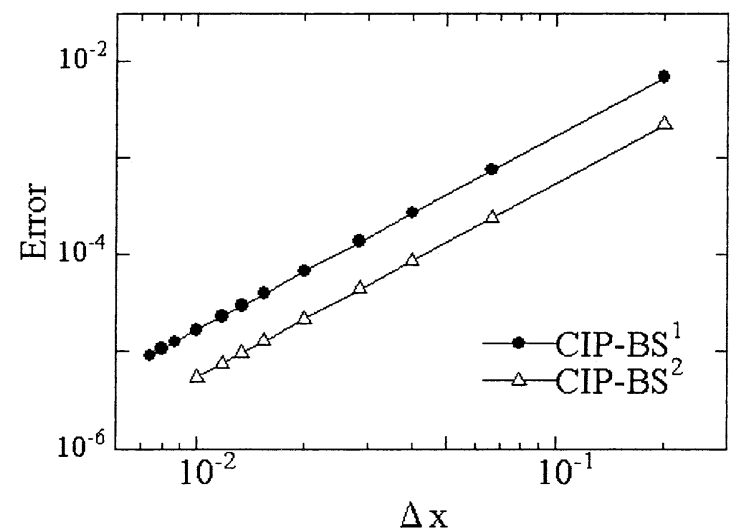

(c)

Fig. 7 Numerical solution of the Poisson equation $\frac{\partial^{2} f}{\partial x^{2}}=\delta(x)$. (a),(b) Profile and the deviation from the exact solution at each grid point by the CIP-BS ${ }^{1}$ method with 11 and 101 grid points, respectively. (c) The deviation from the exact solution versus the grid interval by the CIP-BS ${ }^{1}$ and CIP-BS ${ }^{2}$ method

$$
\begin{aligned}
= & \frac{9 \Delta x}{70} g_{n-1}^{0}+\frac{26 \Delta x}{35} g_{n}^{0}+\frac{9 \Delta x}{70} g_{n+1}^{0}+\frac{13 \Delta x^{2}}{420} g_{n-1}^{1} \\
& -\frac{13 \Delta x^{2}}{420} g_{n+1}^{1} \\
- & \frac{1}{10} f_{n-1}^{0}+\frac{1}{10} f_{n+1}^{0}+\frac{\Delta x}{30} f_{n-1}^{1}-\frac{4 \Delta x}{15} f_{n}^{1}+\frac{\Delta x}{30} f_{n+1}^{1} \\
= & -\frac{13 \Delta x^{2}}{420} g_{n-1}^{0}+\frac{13 \Delta x^{2}}{420} g_{n+1}^{0}-\frac{\Delta x^{3}}{140} g_{n-1}^{1}+\frac{2 \Delta x^{3}}{105} g_{n}^{1}
\end{aligned}
$$

$$
-\frac{\Delta x^{3}}{140} g_{n+1}^{1},
$$

where $g_{i}^{k}=<\phi_{k, i} \mid g>$, as

$$
\begin{aligned}
f^{\prime \prime} & +\frac{1}{15} f^{(4)} \Delta x^{2}+\frac{1}{600} f^{(6)} \Delta x^{4}+O\left(x^{6}\right) \\
= & g+\frac{1}{15} g^{\prime \prime} \Delta x^{2}+\frac{1}{2520} g^{(4)} \Delta x^{4}+O\left(x^{6}\right),
\end{aligned}
$$

for the CIP-BS ${ }^{1}$ method. In the same way, we obtain

$$
\begin{aligned}
f^{\prime \prime}+ & \frac{5}{84} f^{(4)} \Delta x^{2}+\frac{1}{720} f^{(6)} \Delta x^{4}+\frac{1}{52920} f^{(8)} \Delta x^{6}+O\left(x^{8}\right) \\
= & g+\frac{5}{84} g^{\prime \prime} \Delta x^{2}+\frac{1}{720} g^{(4)} \Delta x^{4}+\frac{31}{1108800} g^{(6)} \Delta x^{6} \\
& +O\left(x^{8}\right),
\end{aligned}
$$

for the CIP-BS ${ }^{2}$ method. Again, in Eqs. (22) and (23), the functions $f, g$ are seen to be functions of the form $h+A h^{\prime \prime} \Delta x^{2}, h+A h^{\prime \prime} \Delta x^{2}+B h^{(4)} \Delta x^{4} \quad(h=f$ or $g$ ), respectively. Therefore, if the function $g$ is continuous, the CIP-BS ${ }^{1}$ and CIP-BS ${ }^{2}$ method are about fourth and sixth order accuracy, respectively. However, if the function $g$ is $\delta(x)$, this is approximated as $\phi_{0,0}(x)$ by Eq. (1), i.e. $\lim _{\Delta x \rightarrow 0}\left(\phi_{0,0}(x) / \Delta x\right)=\delta(x)$. Then Eqs. (22) and (23) become $f^{\prime \prime}+O\left(\Delta x^{2}\right)=g$ and both the CIP-BS ${ }^{1}$ and CIP-BS ${ }^{2}$ method show second order accuracy as depicted in Fig. 7 (c).

\section{Conclusion}

We have solved typical parabolic, hyperbolic, and elliptic partial differential equations with high accuracy using the CIP-BS method. It is shown that the CIP-BS ${ }^{1}$ method has about fourth order accuracy, and the CIP-BS ${ }^{2}$ method improves accuracy by about two orders. This is a direct consequence of the fact that the expansion Eq. (1) can represent exact solution with any degree of accuracy not only in the limit $N \rightarrow \infty$ but also $K \rightarrow \infty$.

For the thermal diffusion equation and advection equation, we have explicitly shown that there are conservative quantities consistent with analytical requirements. The degree of conservation depends on the accuracy of the integration method we adopt to time integrate the discretized differential equations. As for the Poisson equation, we have proven that the CIP-BS method can solve problems which have a singular point where the spatial derivative is not continuous, and shown the tendency that numerical errors accumulate near the singular point.

We have indicated the effectiveness of the CIP-BS method for solving typical one-dimensional partial differential equations. The method is straightforwardly applicable to complex physical and engineering problems.

\section{Acknowledgments}

The authors would like to express heartfelt thanks to Prof. T. Kunugi at Kyoto University, Prof. T. Yabe and Prof. T. Aoki at Tokyo Institute of Technology for their valuable discussion and encouragement. In addition, we 
thank Dr. J. Koga at Japan Atomic Energy Research Institute for his careful reading of our manuscript.

\section{Appendix}

Table 1 Scalar products for the CIP-BS ${ }^{0}$ method

\begin{tabular}{lccc}
\hline & $n^{\prime}=n-1$ & $n^{\prime}=n$ & $n^{\prime}=n+1$ \\
\hline$<\phi_{0, n} \mid \phi_{0, n^{\prime}}>$ & $\Delta x / 6$ & $2 \Delta x / 3$ & $\Delta x / 6$ \\
$<\phi_{0, n}\left|D_{x}\right| \phi_{0, n^{\prime}}>$ & $-1 / 2$ & 0 & $1 / 2$ \\
$<\phi_{0, n}\left|D_{x}^{2}\right| \phi_{0, n^{\prime}}>$ & $1 / \Delta x$ & $-2 / \Delta x$ & $1 / \Delta x$ \\
\hline
\end{tabular}

Table 2 Scalar products for the CIP-BS ${ }^{1}$ method

\begin{tabular}{lccc}
\hline & $n^{\prime}=n-1$ & $n^{\prime}=n$ & $n^{\prime}=n+1$ \\
\hline$<\phi_{0, n} \mid \phi_{0, n^{\prime}}>$ & $9 \Delta x / 70$ & $26 \Delta x / 35$ & $9 \Delta x / 70$ \\
$<\phi_{0, n} \mid \phi_{1, n^{\prime}}>$ & $13 \Delta x^{2} / 420$ & 0 & $-13 \Delta x^{2} / 420$ \\
$<\phi_{1, n} \mid \phi_{0, n^{\prime}}>$ & $-13 \Delta x^{2} / 420$ & 0 & $13 \Delta x^{2} / 420$ \\
$<\phi_{1, n} \mid \phi_{1, n^{\prime}}>$ & $-\Delta x^{3} / 140$ & $2 \Delta x^{3} / 105$ & $-\Delta x^{3} / 140$ \\
$<\phi_{0, n}\left|D_{x}\right| \phi_{0, n^{\prime}}>$ & $-1 / 2$ & 0 & $1 / 2$ \\
$<\phi_{0, n}\left|D_{x}\right| \phi_{1, n^{\prime}}>$ & $-\Delta x / 10$ & $\Delta x / 5$ & $-\Delta x / 10$ \\
$<\phi_{1, n}\left|D_{x}\right| \phi_{0, n^{\prime}}>$ & $\Delta x / 10$ & $-\Delta x / 5$ & $\Delta x / 10$ \\
$<\phi_{1, n}\left|D_{x}\right| \phi_{1, n^{\prime}}>$ & $\Delta x^{2} / 60$ & 0 & $-\Delta x^{2} / 60$ \\
$<\phi_{0, n}\left|D_{x}^{2}\right| \phi_{0, n^{\prime}}>$ & $6 / 5 \Delta x$ & $-12 / 5 \Delta x$ & $6 / 5 \Delta x$ \\
$<\phi_{0, n}\left|D_{x}^{2}\right| \phi_{1, n^{\prime}}>$ & $1 / 10$ & 0 & $-1 / 10$ \\
$<\phi_{1, n}\left|D_{x}^{2}\right| \phi_{0, n^{\prime}}>$ & $-1 / 10$ & 0 & $1 / 10$ \\
$<\phi_{1, n}\left|D_{x}^{2}\right| \phi_{1, n^{\prime}}>$ & $\Delta x / 30$ & $-4 \Delta x / 15$ & $\Delta x / 30$ \\
\hline
\end{tabular}

Table 3 Scalar products for the CIP-BS ${ }^{2}$ method

\begin{tabular}{lccc}
\hline & $n^{\prime}=n-1$ & $n^{\prime}=n$ & $n^{\prime}=n+1$ \\
\hline$<\phi_{0, n} \mid \phi_{0, n^{\prime}}>$ & $25 \Delta x / 231$ & $181 \Delta x / 231$ & $25 \Delta x / 231$ \\
$<\phi_{0, n} \mid \phi_{1, n^{\prime}}>$ & $151 \Delta x^{2} / 4620$ & 0 & $-151 \Delta x^{2} / 4620$ \\
$<\phi_{0, n} \mid \phi_{2, n^{\prime}}>$ & $181 \Delta x^{3} / 55440$ & $281 \Delta x^{3} / 27720$ & $181 \Delta x^{3} / 55440$ \\
$<\phi_{1, n} \mid \phi_{0, n^{\prime}}>$ & $-151 \Delta x^{2} / 4620$ & 0 & $151 \Delta x^{2} / 4620$ \\
$<\phi_{1, n} \mid \phi_{1, n^{\prime}}>$ & $-19 \Delta x^{3} / 1980$ & $104 \Delta x^{3} / 3465$ & $-19 \Delta x^{3} / 1980$ \\
$<\phi_{1, n} \mid \phi_{2, n^{\prime}}>$ & $-13 \Delta x^{4} / 13860$ & 0 & $13 \Delta x^{4} / 13860$ \\
$<\phi_{2, n} \mid \phi_{0, n^{\prime}}>$ & $181 \Delta x^{3} / 55440$ & $281 \Delta x^{3} / 27720$ & $181 \Delta x^{3} / 55440$ \\
$<\phi_{2, n} \mid \phi_{1, n^{\prime}}>$ & $13 \Delta x^{4} / 13860$ & 0 & $-13 \Delta x^{4} / 13860$ \\
$<\phi_{2, n} \mid \phi_{2, n^{\prime}}>$ & $\Delta x^{5} / 11088$ & $\Delta x^{5} / 4620$ & $\Delta x^{5} / 11088$ \\
$<\phi_{0, n}\left|D_{x}\right| \phi_{0, n^{\prime}}>$ & $-1 / 2$ & 0 & $1 / 2$ \\
$<\phi_{0, n}\left|D_{x}\right| \phi_{1, n^{\prime}}>$ & $-11 \Delta x / 84$ & $11 \Delta x / 42$ & $-11 \Delta x / 84$ \\
$<\phi_{0, n}\left|D_{x}\right| \phi_{2, n^{\prime}}>$ & $-\Delta x^{2} / 84$ & 0 & $\Delta x^{2} / 84$ \\
$<\phi_{1, n}\left|D_{x}\right| \phi_{0, n^{\prime}}>$ & $11 \Delta x / 84$ & $-11 \Delta x / 42$ & $11 \Delta x / 84$ \\
$<\phi_{1, n}\left|D_{x}\right| \phi_{1, n^{\prime}}>$ & $13 \Delta x^{2} / 420$ & 0 & $-13 \Delta x^{2} / 420$ \\
$<\phi_{1, n}\left|D_{x}\right| \phi_{2, n^{\prime}}>$ & $13 \Delta x^{3} / 5040$ & $\Delta x^{3} / 504$ & $13 \Delta x^{3} / 5040$ \\
$<\phi_{2, n}\left|D_{x}\right| \phi_{0, n^{\prime}}>$ & $-\Delta x^{2} / 84$ & 0 & $\Delta x^{2} / 84$ \\
$<\phi_{2, n}\left|D_{x}\right| \phi_{1, n^{\prime}}>$ & $-13 \Delta x^{3} / 5040$ & $-\Delta x^{3} / 504$ & $-13 \Delta x^{3} / 5040$ \\
$<\phi_{2, n}\left|D_{x}\right| \phi_{2, n^{\prime}}>$ & $-\Delta x^{4} / 5040$ & 0 & $\Delta x^{4} / 5040$ \\
$<\phi_{0, n}\left|D_{x}^{2}\right| \phi_{0, n^{\prime}}>$ & $10 / 7 \Delta x$ & $-20 / 7 \Delta x$ & $10 / 7 \Delta x$ \\
$<\phi_{0, n}\left|D_{x}^{2}\right| \phi_{1, n^{\prime}}>$ & $3 / 14$ & 0 & $-3 / 14$ \\
$<\phi_{0, n}\left|D_{x}^{2}\right| \phi_{2, n^{\prime}}>$ & $\Delta x / 84$ & $-\Delta x / 42$ & $\Delta x / 84$ \\
$<\phi_{1, n}\left|D_{x}^{2}\right| \phi_{0, n^{\prime}}>$ & $-3 / 14$ & 0 & $3 / 14$ \\
$<\phi_{1, n}\left|D_{x}^{2}\right| \phi_{1, n^{\prime}}>$ & $\Delta x / 70$ & $-16 \Delta x / 35$ & $\Delta x / 70$ \\
$<\phi_{1, n}\left|D_{x}^{2}\right| \phi_{2, n^{\prime}}>$ & $\Delta x^{2} / 210$ & 0 & $-\Delta x^{2} / 210$ \\
$<\phi_{2, n}\left|D_{x}^{2}\right| \phi_{0, n^{\prime}}>$ & $\Delta x / 84$ & $-\Delta x / 42$ & $\Delta x / 84$ \\
$<\phi_{2, n}\left|D_{x}^{2}\right| \phi_{1, n^{\prime}}>$ & $-\Delta x^{2} / 210$ & 0 & $\Delta x^{2} / 210$ \\
$<\phi_{2, n}\left|D_{x}^{2}\right| \phi_{2, n^{\prime}}>$ & $-\Delta x^{3} / 1260$ & $-\Delta x^{3} / 315$ & $-\Delta x^{3} / 1260$ \\
\hline & & & \\
& & &
\end{tabular}

\section{References}

( 1 ) Yabe, T. and Aoki, T., A Universal Solver for Hyperbolic Equations by Cubic-Polynomial Interpolation. I One-Dimensional Solver, Comput. Phys. Commun., Vol.66 (1991), pp.219-232.

( 2 ) Yabe, T., Ishikawa, T., Wang, P.Y., Aoki, T., Kadota, Y. and Ikeda, F., A Universal Solver for Hyperbolic Equations by Cubic-Polynomial Interpolation. II Two-and Three-Dimensional Solvers, Comput. Phys. Commun., Vol.66 (1991), pp.233-242.

( 3 ) Yabe, T. and Wang, P.Y., Unified Numerical Procedure for Compressible and Incompressible Fluid, J. Phys. Soc. Japan, Vol.60 (1991), pp.2105-2108.

( 4 ) Yabe, T., Simulation of Laser-Induced Melting and Evaporation Dynamics by the Unified Solver CIP for Solid, Liquid and Gas, Mathematical Modeling of Weld Phenomena 4, edited by Cerjak, H., (1998), Cambridge Univ. Press, Cambridge, U.K.

( 5 ) Yabe, T. and Xiao, F., Simulation Technique for Dynamical Evaporation Processes, Nucl. Eng. and Design, Vol.155 (1995), pp.45-53.

(6) Yabe, T. and Xiao, F., Description of Complex and Sharp Interface with Fixed Grids in Incompressible and Compressible Fluid, Comput. Math. Applic., Vol.29 (1995), pp.15-25.

( 7 ) Yabe, T., Xiao, F. and Utsumi, T., Constrained Interpolation Profile Method for Multiphase Analysis, J. Comput. Phys., Vol.169 (2001), pp.556-593.

( 8 ) Utsumi, T., Yabe, T., Koga, J., Aoki, T. and Sekine, M., Accurate Basis Set by the CIP Method for the Solutions of the Schrödinger Equation, Comput. Phys. Commun., Vol.157 (2004), pp.121-138.

(9) Aoki, T., Interpolated Differential Operator (IDO) Scheme for Solving Partial Differential Equations, Comput. Phys. Commun., Vol.102 (1997), pp.132-146.

(10) Press, W.H., Tuekolsky, S.A., Vettering, W.T. and Flannery, B.P., Numerical Recipes in Fortran 77, (1986), Cambridge Univ. Press.

(11) Hindmarsh, A.C., LSODE and LSODIS, Two New Initial Value Ordinary Differential Equation Solvers, ACM-SIGNUM Newslett., Vol.15 (1980), pp.10-11. 